\title{
LA FORMA PRONOMINAL LATINA IPSE: SU ORIGEN
}

In opposition to those interpretations which consider the element -pse of the pronoun ipse as an emphatic particle with little regard for the question of its origin, and those others which see a particular semantic value in this particle, the author of this article suggests that the pronominal form ipse is the result of a generalization of -pse to the whole pronominal paradigm beginning with eumpse. This pronoun is characterised by an exclusively emphatic function, where the presence of an epenthetic $p$ in the sequence *eum-se > eumpse is phonetically legitimised.

1. El pronombre latino ipse contiene en su estructura formal un segmento fónico - pse sobre cuyo origen y valor los estudiosos han expresado opiniones diversas. La verdad es que un tanto por ciento muy elevado de las propuestas expresadas en torno a este problema apuntan a consideran al elemento - pse una partícula de carácter enfático que servía para reforzar al pronombre. Dicha partícula encontraría un paralelismo formal y semántico en otras como -pte, -pe, -dem, etc. Por otro lado, este pronombre ha sufrido remodelaciones posteriores llegando a presentar un paradigma idéntico al tipo regular bonus, $-a,-u m$, regularización cuya explicación sin duda se encuentra en el latín popular'. Nuestro trabajo pretende sencillamente tratar de dar una explicación a esta forma sobre una base fonética y dentro del conjunto estructural representado por otros pronombres cuya formación y valores están interrelacionados con ipse. Así sucede con iste e ille entre otros ${ }^{2}$.

2. Dejando a un lado el paradigma canónico de ipse, quienes han querido ver en -pse una partícula autónoma de refuerzo presentan una serie de datos residuales que no aparecen en el paradigma canónico, así:

I Ya desde Plauto asistimos a la tematización del pronombre ipse. Así ipsus en Trin. 985, 988 en donde también leemos ipsissimus, en Ter. Phormio 187 frente a 196 donde aparece ipsest. La forma ipsi de gen. sg. está documentada en Afranio 228 (citado por A. López López 1983).

2 Para un estudio de los valores y funciones de estos pronombres puede consultarse S. Mariner 1968, pp. 131-143. 
-nom. sg. f. eapse PF 68, PI. Cas. 604, Curc. 161, Truc. 24.

-ac. sg. f. eampse Pl. Cist. 170, Poen. 272.

-abl. sg. f. eāpse Pl. Curc. 534, Trin. 974; la forma adverbial reāpse PF 348 (reapse est re ipsa).

-nom. pl. f. eaepse PI. Pseud. 833

-ac. sg. m. eumpse PI. Pers. $603^{3}$

-abl. sg. m. eōpse Pl. Curc. 538

Sabemos que el paradigma comúnmente empleado y que anteriormente hemos definido como canónico se caracteriza por una generalización consistente en mantener el primer miembro indeclinable $(i-$ a lo largo de todo el paradigma) en tanto que el segundo miembro estaría sujeto al proceso de la declinación ${ }^{4}$. Ahora bien en un primer estadio el elemento declinado sería el primero en tanto que el segundo permanecería indeclinable y prueba de ello serían los ejemplos arriba mencionados 5 .

Se ha querido ver entre ambos estadios una fase intermedia consistente en presentar ambos elementos declinados. Sin embargo las formas aducidas para propugnar dicha fase intermedia tropiezan con dificultades ya que eapsa de Pl. Cas. 602 nom. sg. f. aparece en los mejores manuscritos como eapse. Otro tanto sucede con eōpso Pl. Curc. 538, que en otros manuscritos aparece como eōpse e igualmente con formas como eumpsum que es sin duda eumpse Pl. Truc. 114, Caecil. 26, Pac. 48.

También tenemos atestiguadas otras formas análogas a partir del tema *so, "sā tales como sapsa Enn. Ann. 406, Pac. 372, sumpse Pl. Truc. 159. Pero no tenemos, salvo sapsa, posible remodelación, ningún ejemplo en que aparezca el estadio con ambos elementos declinados ${ }^{6}$.

3. Para un grupo de estudiosos el pronombre ipse encuentra su explicación en la adición al elemento pronominal $i$ - de una serie de partículas. Según Molina Yébenes ${ }^{7}$ tendríamos ${ }^{*} i+{ }^{*} p e$ (que vemos en nem-pe, quip-pe, quis-p-iam) + "se, partícula sobre la que se creó la flexión del reflexivo de tercera persona. Parecida es la propuesta de Pisa-

${ }^{3}$ Las formas eumpsum non eampsam de PI. Truc. 133 no son de tradición segura. Así lo afirma M. Leumann 1963, p. 286.

4 Procedimiento que también alcanzó a iste e ille pero no a idem ni a hic.

3 La forma apse citada por Consencio, cf. H. Keil 1961, p. 396, al decir de F. Sommer 1914, p. 432 está falsamente obtenida por reapse.

- No aparecen atestiguadas formas como sumpswm < "som-som ni sampsam < *sām-sām.

TJ. Molina Yébenes 1968, p. 163. 
$\mathrm{ni}^{8}$ que parte de ${ }^{*} i+p e+s o$. El análisis último al que llega Kieckers ${ }^{9}$ discurre también por idénticos caminos al ver en ipse el resultado de *is-pse con caida disimilatoria de la primera silbante. A su vez -pse posiblemente procedería, según este autor, de *-p(e)-se donde se guardaría una relación con *so y en definitiva la flexión de ipse iría en asonancia con la de iste e ille. Unicamente su nom.-ac. neutro del sg. seguiría la declinación nominal en -um frente a $-u d$ de los otros pronombres.

4. La opinión comúnmente aceptada, sin embargo, es la que ve en -pse una partícula indeclinable y dotada de un valor de refuerzo ${ }^{10}$. Sommer recordando una antigua propuesta de Kretschmer según la cual la partícula -pse estaría relacionada con el ac. sicil.-dórico $\psi \varepsilon \varepsilon=$ $\sigma \phi \varepsilon$, propugna como formas antiguas *is-pse y eumpse de modo que el esquema normalizado ipse ipsum sería secundario según ille e iste al tiempo que una forma como eapsa sería simplemente una fórmula de compromiso de los dos paradigmas anteriores. Al mismo tiempo y siguiendo el análisis de Sommer ", a partir de una forma antigua como sapsa ${ }^{12}$ podría pensarse en un ac. "sampsam $=$ *sām-sām, forma redoblada como sē-sē y em-em. A partir de sampsam < *sām-sām se formó un nominativo sapsa y correspondiendo al acusativo *som-som > sompsom habriamos esperado *so(s)psos temático o *sopse atemático. Como so- en esta forma fue desplazado por is, se crearon eapsa, ${ }^{*} i(s) p$ sus e ${ }^{*} i(s) p s e$. No obstante debemos poner de manifiesto que la forma eapsa es muy discutible y los mejores manuscritos presentan eapse a la vez que formas como *sampsam y *sompsom no están atestiguadas del mismo modo que lecturas como eumpsum Pl. Truc. 114 y eampsam Truc. 133 deben leerse como eumpse y eampse respectivamente.

Ya dijimos anteriormente que la partícula -pse era comúnmente identificada como una partícula de refuerzo análoga a -pte. Partiendo de esta afirmación se ha querido ver la forma ipse como un elemento cuya flexión tendría como modelo a ille de suerte que únicamente el

\footnotetext{
${ }^{8}$ V. Pisani 1945, p. 78. Cf. también M. Leumann 1963, p. 289.

' E. Kieckers 1962 II, p. 148 donde a pesar de todo afirma: «Die Etymologie von ipse ist unsicher".

10 Esta es la escueta explicación que hallamos en R. G. Kent 1945, p. 130; J. Safarewicz 1969, p. 181; A. Ernout 1953, p. 96; P. Monteil 1970, p. 233:-pse sería una partícula invariable mal establecida que marcaba al igual que -dem un matiz de insistencia; A. Meillet-J. Vendryes 1968, p. 496. En idénticos términos se manifiesta M. Leumann 1963, p. 128.

"Este alambicado análisis de la forma ipse puede verse en J. Sommer 1914, p. 431, y en $K E, 1914$, pp. 118-119.

${ }_{12}$ Sapsa está atestiguada en Enn. Ann. 406; Pac. 372 (citados por E. H. Warmington 1967).
} 
segmento terminal -pse se declinaba. La partícula -pse según ErnoutMeillet no se encuentra fuera del Itálico y estos autores ven en el contraste nom. sg. ipse y abl. sg. eōpte una huella de un antiguo estado de cosas que respondería a la antigua oposición del sánscrito que presenta sa- para el nom. sg. $\mathrm{m}$. y f. y ta-para el resto de la declinación ${ }^{13}$.

No han faltado autores que han sugerido una relación entre hitita pat 'mismo' y el latín -pse, -pte, -pot- (potis) ${ }^{14}$. Fue E. Benveniste quien hizo un escueto análisis de esta última sugerencia en un artículo dedicado a los problemas semánticos de la reconstrucción al observar la existencia de una homofonía entre la raíz "pet-/pot( $i$ ) 'jefe' y una partícula "pet/pot(i) de identidad que significa 'mismo' y que aparece en hit. -pet, av. -paiti, lat. -pte, lit. -pat ${ }^{15}$.

A las propuestas hasta ahora enunciadas viene a sumarse últimamente el análisis de Otrębsky quien, después de desechar como incorrecta la relación de las partículas -pse y -pte ya que la segunda sería la unión de dos partículas -pe y $-t e^{16}$ y tras afirmar la diferencia de usos de ambas en época antigua, señalaba que junto a las formas pronomi-

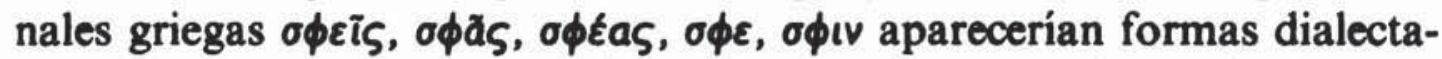
les con $\psi$ por $\sigma \phi: ~ \psi \varepsilon$ y $\psi i v$ de modo que el pronombre reflexivo ide. *sebh- $\left(<{ }^{*}\right.$ se-bhe $)$ aparecería oponiéndose al grado cero *sbh-, de aquí formas como aesl. sebe / gr. $\sigma \phi \varepsilon i \bar{\zeta}$. Ahora bien *se y *bhe pudieron sufrir una metátesis locativa de manera que junto a *sbh-pudo aparecer *bhes- y junto a *sbh- pudo darse *bhs- que daría *ps-. El morfema "bhes- significaria en un principio 'reunión' y tal raíz se atestiguaría en palabras como ai. sabhā- 'reunión', aesl. beséda 'coloquio, discurso' ${ }^{17}$. En parecidos términos, aunque de una forma más concisa, se había

${ }^{13}$ Cf. A. Ernout-A. Meillet 1967 , p. 322, quienes insisten en que la partícula -pse está compuesta de - $p$ - (como en -p-te) y -se que recuerda a la partícula augens del irlandés.

14 H. Pedersen 1937, p. 77 ss.; H. Kronasser 1956, p. 50.

is Si entre ambas raíces hubo una relación semántica, habría que preguntarse cuál de ellas sería el punto de partida. Las respuestas han sido de signo diverso. Benveniste opina que formas como aúrós o ipse nunca llegaron a significar 'jefe (de la casa)' como pretendía Pedersen. A lo sumo, en determinados contextos podría haber tenido una acepción de este tipo pero en un nivel familiar, no alcanzando dicha acepción fuera de estos contextos. Cf. E. Benveniste 1954, p. 260.

16 J. Otząbsky 1966, p. 19.

17 J. Otrapsky 1966 , p. 20 , quien propugna a partir de su teoría que las formas latinas sum-pse, sam-psam, etc., no deben retrotraerse a raices ide. "so-, sā- sino que tendrían su origen en ipsum, ipsam con formas sin $i$ - de época antigua, apareciendo "psum, "psam > sum, sam con caída de $p$ iniciat en grupos formados por $p s-$ M. Lejeune 1955 , p. 63 señala que los poetas siracusanos escriben por interversión de sus elementos $\psi i v, \psi \varepsilon$ por $\sigma \phi i v, \sigma \phi \varepsilon$. 
pronunciado Chantraine, quien a pesar de todo sigue considerando las formas pronominales griegas poco diáfanas ${ }^{18}$.

5. En resumen, los intentos de explicación de la partícula -pse del pronombre latino se reducen a dos:

a) -pse es una partícula de refuerzo autónoma en la que algunos han querido ver la adición de dos partículas más elementales, a saber, pe y se.

b) La partícula latina -pse estaría en principio dotada de un valor semántico 'mismo' en relación con hit. pat, av. -paiti, etc... o bien con la raiz del pronombre reflexivo *se-bhe cuyo grado cero *sbhe tras un proceso de metátesis locativa de las consonantes daría *bhse $>$ pse, fenómeno que hallaría su paralelo en formas griegas como $\psi \iota v$ y $\psi \varepsilon$ frente a $\sigma \phi \imath v$ y $\sigma \phi \varepsilon$.

6. Frente a estas propuestas pretendemos presentar una explicación, a nuestro juicio, más sencilla y coherente.

Sabemos que una buena parte de las raíces pronominal-adverbiales estaban constituidas por una consonante más la vocal $e(\mathrm{Ce})$ y que dichas formas terminaron o bien adoptando una flexión teniendo por modelo la flexión nominal, especialmente los temas en $-o$ y $-\bar{a}$ que ya se habrian especializado en la noción de género masculino y femenino respectivamente, o bien permaneciendo en su forma más antigua, es decir, $\mathrm{Ce}$, se habían añadido a otros temas también pronominales sirviendo como partículas de refuerzo al tiempo que fortalecían su sustancia fónica. Así a partir de *se se crearon *so, *sā; a partir de *te tenemos *to, * $t \bar{a}$ que encontramos prácticamente en la totalidad de las lenguas indoeuropeas. Pero incluso en latín también podemos percibir las huellas de una antigua flexión cual es el caso de las formas como sum (Ter. Phorm. 1028), sam (Enn. Ann. 219) o bien de formas petrificadas ya como adverbios tales como si < sei loc., tum, tam, dum, nun-c, etc.

Ciertos pronombres, sobre todo aquellos que adquirieron un valor deíctico se reforzaron y para ello añadieron a su forma pronominal otra caracterizada por $\mathrm{Ce}$. Son prueba de ello los pronombres griegos $\boldsymbol{\varepsilon}-\mathrm{k} \boldsymbol{\varepsilon}$ $\varepsilon v-o \varsigma, \delta-\delta \varepsilon, \delta$-ve y en latín hic < ${ }^{*}$ ghi-ke, iste, il-le ${ }^{19}$ y los adverbios $e c$ $c e$, nunc < * nom-ke y la forma ipse, objeto de nuestro estudio.

${ }_{18}$ P. Chantraine 1967 , p. 140 , quien piensa que posiblemente toda la flexión griega tenga su punto de arranque en el dat. $\sigma \phi_{l}<{ }^{*} s-($ en grado cero) $+\phi l$ desinencia de instrumental que vemos por ejemplo en vaó $\phi$. Al lado de $\sigma \phi i$ debió existir una forma de dat. "oфeı que se correspondería con lat. sibi.

19 No está suficientemente claro si para ille debemos partir de la forma adicional *-de, *-ne o *-se. Cf. P. Monteil 1970, pp. 234-235. 
Al pronombre anafórico $i$ - y a su variante en grado pleno $e i$ - se le añadió la vocal temática $o$, de suerte que existieron *io- y *eío-. El primero de estos elementos utilizado en algunas lenguas indoeuropeas para formar los pronombres relativos ${ }^{20}$ no dejó restos en latín que, como es notorio, empleó la forma pronominal ${ }^{*} k^{*} i$-, ${ }^{*} k^{*} o$ - propia de indefinidos e interrogativos para la flexión del relativo. En cambio sí hizo un extenso uso de la formación *eio- como puede apreciarse en el paradigma del anafórico. Es muy posible que en un principio el mero valor referencial del anafórico se expresase mediante la forma $\mathrm{im}^{21}$ a partir de la cual se formaria la forma is de nominativo.

Estas dos formas señalaban las dos funciones fundamentales sintácticas apuntando a los dos polos esenciales del proceso verbal. Cuando a este valor meramente anafórico se le quiso dotar de un valor deíctico o enfático la lengua acudió al expediente de la tematización y al refuerzo mediante partículas de origen adverbial caracterizadas por la estructura $\mathrm{Ce}$, tales como -ke, -de, -ne, -te y -se. La forma de acus. sg. m. de un pronombre de este tipo sería *einom-se > eumpse con $p$ anaptíctica ${ }^{22}$ frente al nom. que seria ${ }^{*} i$-se.

Al igual que en los anafóricos, estas dos formas representarian un papel esencial en las funciones sintácticas. A partir de eumpse se formaría el nominativo ipse al ser analizada -pse como una partícula cuya jus-

${ }^{20}$ A título de ejemplo podemos citar ai. yás, yá, yád o el gr. $8 \varsigma, \hbar, 8$.

21 También está atestiguada la forma em en las Doce Tablas.

22 En latín la $p$ epentética se produjo en las secuencias *-ms- > -mps-, *-mt- > $-m p t-,{ }^{*}-m l->-m p l-$. M. Niedermann 1953, pp. 152-153 matizaba que formas como

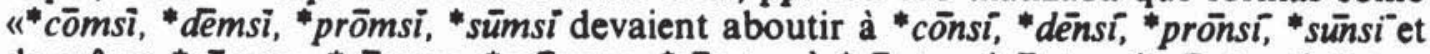
de même "cōmtus, "dèmtus, "prōmtus, "sūmtus à *cōntus, "dêntus, "prōntus, "sūntus, mais pour des raisons d'ordre étymologique l'm a été rétabli dans toutes ces formes". Siguiendo a Niedermann vemos a M. Bassols, 1967, p. 218. Cf. también J. Molina Yébenes 1968, pp. 67-68; P. Monteil 1970, p. 81; A. Maniet 1975, p. 84; A. Meillet-J. Vendryes 1968, pp. 83 y 85; J. Safarewicz 1969, pp. 113-114; E. Kieckers 1962, pp. 131, 141, 143; F. Sommer 1914, pp. 228, 237, 244, 245; C. Juret 1921, p. 182. Se ha querido ver en la forma osco-umbra esuf una forma paralela a ipse en donde la $s$ medial del osco-umbro sería el resultado del grupo *-ps-. Pero esta propuesta no es comúnmente aceptada por cuanto muchos autores consideran la forma esuf como un adverbio formalmente equiparable a otros como o. PUf, pel. ecuf. Ya R. von Planta vio en o. essuf y u. esuf un adverbio < *ekso- < *ekeso. Cf. R. von Planta 1892-97 I, p. 378. Para C. D. Buck 1928, pp. 72-73, 79, 143 la forma es(s) uf significaría ipse, procediendo de *eps $\delta$-ns. Sin embargo termina el estudio confesando que la cuestión en su conjunto es problemática. Identifican con ipse la forma es $(u) f G$. Bottiglioni 1954, pp. 74, 127; J. W. Poultney 1959, pp. 110-111 y 187-188; G. Devoto 1954 , pp. 108, 112; F. Bücheler 1883, pp. 6, 9, 138; F. Muller 1926, p. 221. E. Vetter 1953 tiene sus dudas y normalmente lo interpreta como una forma adverbial, así en pp. 192, 200 traduciéndolo por «eo» y pp. 209, 217 como «ibi». 
tificación fonética sólo se encuentra en el acus. sg. ${ }^{23}$. La partícula -pse a partir de aquí se generalizó en el resto de la flexión como vimos anteriormente.

Es cierto que se no aparece como forma pronominal autónoma en latín ni tampoco como partícula añadida a una forma pronominal (excepto el controvertido caso de ille < *-se, *-de, *-ne?). A pesar de ello puede ser verosímilmente reconstruida por medio de una sencilla proporción, a saber: tum: te; dum: de; quom: -que = som, sām, sōs, sei: ${ }^{*}$ se. Por otro lado la partícula se se encuentra en otras lenguas indoeuropeas bien como elemento autónomo o bien como forma añadida a un tema pronominal. Así en Tocario B encontramos se, sa, te correspondiendo al griego $\delta, \tilde{\eta}, \tau^{24}$. En antiguo sajón aparece se como nom. sg. funcionando como pronombre y como artículo ${ }^{25}$. Como partícula añadida a un tema encontramos se (junto a so y $s \bar{a}$ ) en antiguo irlandés en el sintagma nombre-artículo creando adjetivos demostrativos ${ }^{26}$.

Pero el latín dio un paso más en el tratamiento morfológico de ipse. Trasladó la flexión del primer elemento al segundo de modo que ahora la partícula -pse iba a recibir la flexión canónica propia de adjetivos del tipo bonus, $-a,-u m$ excepto en el genitivo, dativo y nominativo sg. que seguiria siendo la forma antigua, e incluso en estos casos el habla popular los asimiló por completo a la flexión normal de los adjetivos ${ }^{27}$.

Idéntico proceso siguieron iste e ille y es curioso observar cómo esta metátesis flexiva se produjo en estos tres pronombres y no tuvo lugar, en cambio, en pronombres como idem o quisque, quidam, etc.

La explicación de este fenómeno posiblemente se encuentre en el hecho de que mientras idem, quisque, etc., tenían usos de carácter fundamentalmente pronominal, en cambio las formas ipse, iste e ille, además de valores pronominales, eran muy frecuentemente empleadas como adjetivos y se vieron irremediablemente influidas por el sistema flexivo nominal.

Se ha querido ver entre ambas fases de flexión un estadio intermedio que se caracterizaría por la flexión simultánea de ambos elementos.

${ }^{23}$ Un desarrollo paralelo hay que suponer para la partícula -pte donde $p$ surgiría por anaptixis a partir del ac. sg. "ejom-te > "eompte. Sin embargo no tenemos en el nominativo sg. *ispte, que seria la forma paralela a ipse, debido por un lado al dificil mantenimiento de un grupo de esta naturaleza y por otro a la generalización de -te tras is-. Cf. sin embargo la forma eopte que sí tenemos atestiguada.

${ }^{24}$ Cf. W. Krause-W. Thomas 1960, p. 164. Cf. también F. R. Adrados 1975, p. 819.

${ }^{25}$ Cf. H. Sweet 1974 , p. 47.

${ }^{26}$ Cf. R. Thurneysen $1966_{2}$, p. 299 ss.

${ }^{27}$ Cf. ipsus PI. Pseud. 1142; Ter. Hecyra 455; ipsi Afranio 228; ipsō dat. en Apul. Met. X 10. 
Es decir, entre eumpse e ipsum se ha postulado un estadio intermedio *eumpsum. Sin embargo, como apuntamos ya antes no tenemos datos fidedignos para afirmar que en latín se hubiese dado esta fase intermedia por cuanto los ejemplos aportados tales como eapsa Pl. Cas. 602; eumpsum Pl. Truc. 114; eampsam Pl. Truc. 113 aparecen en otros manuscritos más fiables como eapse, eumpse y eampse respectivamente.

7. En definitiva podemos concluir que frente a las propuestas que analizan -pse como una partícula de refuerzo pero sin adentrarse en el proceso de su génesis y aquellas otras que pretenden ver en esta partícula un valor semántico, en ocasiones producto de complicadas metátesis de fonemas, la forma pronominal ipse es el resultado de una generalización de -pse a todo el paradigma pronominal a partir del caso ac. sg. eumpse donde está legitimada fonéticamente la aparición de una $p$ epentética en la secuencia ${ }^{*}$ eum-se $>$ eumpse ${ }^{28}$.

Así pues, a partir de la base pronominal ei-caracterizada por la vocal temática y flexionada en caso acusativo, forma a la que se añadiría la partícula *se, paralela a otras como -ke, -de, -te que también aparecen en otros pronombres, tendríamos una forma deíctica ${ }^{*} e i$-om-se $>$ eompse $>$ eumpse que tendría en latín una función enfática de exclusividad. En un primer estadio solamente el término constituido por eịo- estaría dotado de flexión y ya a partir de ese momento el elemento -pse sería sentido como partícula y añadido a las demás formas flexivas. En una segunda fase sería la partícula -pse la que recibiría la flexión añadiéndose al término $i$ - que permanecería invariable. No parece en cambio que entre ambos estadios hubiera existido uno intermedio en el que ambos términos recibiesen simultáneamente la flexión.

RAFAel JimEneZ Zamudio

\section{BIBLIOGRAFIA EMPLEADA}

F. R. Adrados 1975: Lingüistica indoeuropea. Madrid.

M. Bassols 1967: Fonética latina. Madrid.

${ }^{28}$ Procesos de generalización de esta naturaleza se producen en todas las lenguas indoeuropeas. Es muy frecuente ver falsos cortes a partir de una forma muy concreta, de suerte que una forma dada cuya justificación fonética o morfológica se encuentra en un contexto también dado puede extenderse fuera de ese contexto. Así nom. sg. en -ēs de *-ēws tiene su origen en el ac. sg. -ēm. En gr. Tíva de ac. sg. es el punto de arranque de formas como tíves, rivwv, tivas, tivos, tívi. En el ai. un ac. sg. del pronombre ayám, iyám, idám que es imám origina formas como el nom. pl. imé, imás, imáni y el ac. pl. imân, imás. 
E. Benveniste 1954: «Problèmes sémantiques de la reconstruction». Word 10, pp. 251-264.

G. Bottiglioni 1954: Manuale dei dialetti italici. Bolonia.

C. D. Buck 1928: A Grammar of Oscan and Umbrian. Boston ${ }_{2}$.

F. Bücheler 1883: Umbrica. Bonn.

P. Chantraine 1967: Morphologie historique du grec. París.

G. Devoto 1954: Tabulae Iguvinae. Roma.

A. Ernout 1953: Morphologie historique du latin. París.

A. Ernout-A. Meillet 1967: Dictionnaire étymologique de la langue latine. París.

C. Juret 1921: Manuel de phonétique latine. París.

H. Keil 1961: Grammatici Latini, vol. V. Hildesheim.

R. G. Kent 1945: The Sounds of Latin. Baltimore .

E. Kieckers 1962: Historische Lateinische Grammatik. Munich.

W. Krause-W. Thomas 1960: Tocharisches Elementarbuch. Heidelberg.

H. Kronasser 1956: Vergleichende Laut- und Formenlehre des Hethitischen. Heidelberg.

M. Lejeune 1955: Traité de phonétique grecque. París $_{2}$

M. Leumann 1963: Lateinische Laut- und Formenlehre. Munich.

A. López López 1983: Fabularum togatarum fragmenta. Salamanca.

S. Mariner 1968: "Contribución al estudio funcional de los pronombres latinos». Actas del III Congreso español de Estudios Clásicos, tomo III. Madrid, pp. 131143.

A. Meillet-J. Vendryes 1968: Traité de grammaire comparée des langues classiques. París.

J. Molina Yébenes 1968: Estudios latinos. I. Barcelona ${ }_{2}$.

P. Monteil 1970: Eléments de phonétique et morphologie du latin. París.

F. Müller 1926: Altitalisches Wörterbuch. Göttingen.

M. Niedermann 1953: Précis de phonétique latine. París.

J. Otrębsky 1966: "Die lateinischen Demonstrativpronomina». Die Sprache 12, pp. 16-25.

H. Pedersen 1937: Hittitisch und die anderen indoeuropäischen Sprachen. Copenhague.

V. Pisani 1945: Lezioni sulla preistoria e storia più antica del latino. Milán.

R. von Planta 1892-1897: Grammatik der oskisch-umbrischen Dialekte I-II. Estrasburgo (= Berlín 1973).

J. W. Poultney 1959: The Bronze Tables of Iguvium. Baltimore.

J. Safarewicz 1969: Historische lateinische Grammatik. Halle.

F. Sommer 1914: Handbuch der lateinischen Laut- und Formenlehre. Heidelberg ${ }_{2,3}$.

F. Sommer KE 1914: Kritische Erläuterungen zur lateinischen Laut- und Formenlehre. Heidelberg.

H. Sweet 1974: Anglo-Saxon Primer. Oxford,

R. Thurneysen 1966: A Grammar of Old Irish. Dublin . $_{2}$

E. Vetter 1953: Handbuch der italischen Dialekte. Heidelberg.

E. H. Warmington 1967: Remains of Old Latin. Harvard. 